\title{
Small Group teaching in clinics - An effective teaching practice for undergraduates
}

\author{
Tapasya Karemore ${ }^{1, *}$, Mukta Motwani ${ }^{2}$, Apeksha Dhole ${ }^{3}$, Anurag Choudhary ${ }^{4}$ \\ ${ }^{1,4}$ Associate Professor, ${ }^{2}$ Professor \& HOD, ${ }^{3}$ Professor, ${ }^{1-4}$ Dept. of Oral Medicine \& Radiology, Vidya Shikshan Prasarak Mandals \\ Dental College and Research Centre, Nagpur, Maharashtra, India
}

*Corresponding Author:

Email: tapasyamds@gmail.com

\begin{abstract}
Aim: To assess student perceptions of small group discussions held during their clinical training, directed towards developing competent health professionals.

Material and Methods: SGT sessions were conducted on each day of posting. The four faculty members included for the project were sensitized to carry out similar SGT sessions for each batch of students as facilitator. Perceptions of the SGT were obtained at the end of posting. Results were drawn with the help of perceptions obtained through validated questionnaire.

Results: The overall response rate was $100 \% .87 \%$ the students felt that objectives for every session were made clear before the sessions. $73 \%$ students said that topics or cases chosen for discussions were appropriate. $93 \%$ agreed that faculty gave them lot of information about the discussed topic. 93\% students commented that what they learned in these sessions was important and will be of use to them in the future.

Conclusion: Students were satisfied with the small group sessions introduced in the department of Oral Medicine and Radiology. Similar SGT sessions can be designed for other subjects in Dentistry in future for better clinical competency.
\end{abstract}

\section{Introduction}

There are many effective methods of teaching, among them; large group didactic lectures and facilitation of open small group teaching are routinely practiced. Small group teaching (SGT) method helps in the development of higher level intellectual skills such as reasoning and problem solving, the development of attitudes and the acquisition of interpersonal skills such as listening, speaking, arguing and group leadership. ${ }^{1}$

For a tutor or facilitator, the core discussion skills required for small group learning are questioning, listening, responding and explaining. These skills from both the ends, builds teamwork and collaborative learning. ${ }^{1,2}$

It is also documented that SGT helps to refresh the knowledge and expertise of faculty and consultants engaged in teaching. It provides guidelines and suggestions on facilitating talking and thinking in groups. ${ }^{3}$

In the subject of Oral Medicine and Radiology, final year students are posted twice in a year (II terms). Clinical postings guide them to learn about variety of cases, their diagnosis and treatment planning. To make this clinical teaching more effective small group teaching sessions were planned for each day of posting.

Aim of the present study was to assess student perceptions of small group discussions held during their clinical training, directed towards developing competent health professionals.

\section{Material and Methods}

After getting approval from IEC, SGT sessions were conducted on each day of posting. The four faculty members included for the project were sensitized to carry out similar SGT sessions for each batch of students as facilitator. The sensitization of faculty to carry out SGT ensured the standardized teaching pattern throughout the year. The SGT sessions were conducted in a same undergraduate section of the department for each batch, which were of 25-35 minutes, each. A structured pattern of SGT was designed to have similar number and types of cases for final year students. Perceptions of the SGT were obtained at the end of posting which means period of five weeks total. Results of the effectivity of SGT was drawn with the help of perceptions obtained through validated questionnaire.

Validated questionnaire was developed which included 17 items. Out of 17, item16 and 17 were open ended while other items were directed to answer with the help of five point likert scale. A mean score against variables was identified and analyzed as percentage distribution.

Students were informed about the SGT feedback well before the sessions. Students were assured on confidentiality of the questionnaire and that no harm or legal consequences will arise regarding the results of the study.

\section{Results}

The study includes 92 final year dental students ( 83 females and 9 males) during 2017-2018 academic years. The overall response rate was $100 \%$. The feedback for routinely planned SGT during clinical postings was obtained under four sections as; A. Structure of small group sessions B. Satisfaction of small group sessions' process, C. Assessment of faculty, and D. Satisfaction or benefits from small group sessions, additionally, suggestions to improve the process and positive points about SGT were also discussed.

a. Structure of small group sessions: $87 \%$ the students felt that objectives for every session were made clear before the sessions and according to $94 \%$ 
students, location and facilities were suitable for the conduction of SGT sessions.

b. Satisfaction of small group sessions' process: $73 \%$ students strongly agree that topics or cases chosen for discussions were appropriate while 3\% ticked that they strongly disagree with this. $68 \%$ students agreed that sessions were thought provoking while 88\% said SGT made them participate in teaching learning process. Self directed learning was learned by $73 \%$ students and $88 \%$ believed that SGT helped them to identify their own learning needs.

c. Assessment of faculty: $77 \%$ students agreed that faculty were enthusiastic in conducting SGT sessions and $93 \%$ felt that faculty gave them lot of information about the discussed topic. $82 \%$ students felt that discussions were planned and were maintained in that manner.

d. Satisfaction or benefits from small group sessions: $93 \%$ students said that what they learned in these sessions was important and will be of use to them in the future. SGT improved ability to think and skills of problem solving of $91 \%$ students.

$73 \%$ students felt that SGT has improved their ability to communicate effectively. Overall $81 \%$ students were satisfied with the quality of these sessions.

Positive stokes obtained through this feedback about SGT in clinical postings were; opportunity to open up with faculty in person so that every query can be discussed, excellent way of co-relating clinical and theoretical learning, it helped to learn patient's perspective and communication skills, it helped to work as a team member and it should be continued ever for undergraduate clinical teaching.

Suggestions to improve SGT sessions were topics selected can be flexible as per students' need and sessions can be longer to discuss theoretical part to some extent.

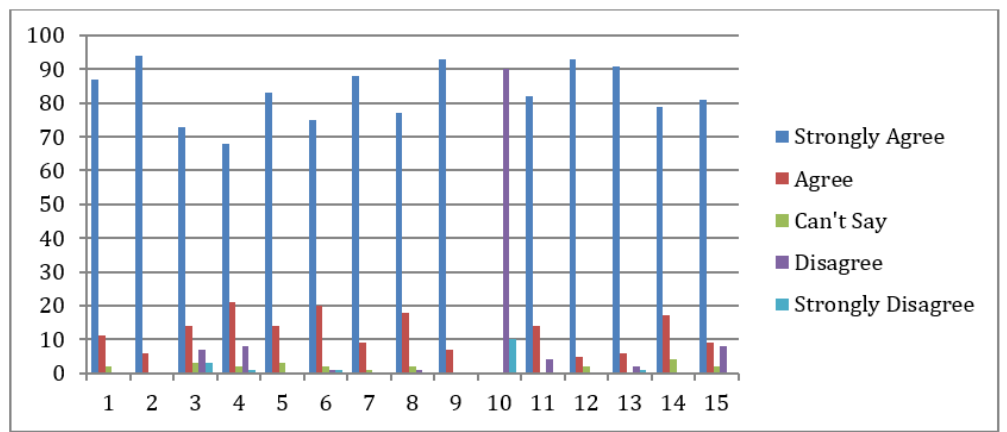

Graph 1: Student's perception about SGT (Axis X- Item, Axis Y- \%)

\section{Discussion}

Small group teaching sessions were always effective in medical teaching. ${ }^{4,5}$ It helps for clinical as well as tutorial learning. Communication and cognitive skills of the tutor and the students are the basis of effective small group learning. ${ }^{1,5}$ In the subject of Oral Medicine and Radiology, during clinical postings, students take variety of cases, learn to diagnose and treat them. This task is challenging for them as they get exposed to clinical scenarios where they are required to deal with patient as expert dental professional which includes everything i.e. right from obtaining case history details to counsel the patient. Looking at the difficulties they face to adapt to this situation after second or third year BDS, the SGT was designed and implemented.

Students are often considered as reliable source of information and have been often used to evaluate the teaching methods and the tutors. ${ }^{1}$ So, this study was planned to get the students' perception of introducing small group learning sessions in the department of Oral Medicine and Radiology.

The overall structure of the sessions, as well as the process of learning was found to be appreciable and successful. Some students find difficulties to be active and critical thinkers. In such circumstances, it was made sure that, the tutors should guide the students to develop their learning skills and decision-making. The tutor should also be a motivator for the students to learn.

The main characteristics of a good tutorial or discussions, as far as tutors are concerned, consist of allowing enough time for discussion, accepting students as partners, refraining from interference and having expertise. ${ }^{4,6}$ Along with the required qualities, the level of enthusiasm among the tutors in the present study was very good. They managed with the challenges like limiting teaching method in the SGT with discussion on clinical cases.

Apart from this, it is commonly observed that mutual discussion in the form of small groups is very effective to clarify and understand the topic under discussion., ${ }^{4,7}$ So, the effectiveness of small group learning sessions can be improved by observing the processes of group interaction too. ${ }^{4,7,8}$ Present study seconds this opinion, as students could learn to interact and discuss the clinical cases amongst them and could demonstrate importance of leadership in group 
dynamics. ${ }^{910}$ Majority of the respondents the topics of discussion as thought provoking and found it helpful in self directed learning too.

Students could also share different cases, clinical scenarios and their knowledge gained from daily postings and resources like books, radiographs, slides, charts and internet etc. ${ }^{4,8,11}$ in SGD. This study revealed the improvement in students' performance in clinical examinations, which again proved the efficiency of SGT in the subject.

In the feedback when asked to comment on areas to improve SGT, few students wanted to discuss theoretical portion of selected topics. This could not be made possible due to posting time limits and was not under the objective, as SGT was planned purely for clinical coaching. Few negative perceptions of the students can be due to different tutors conducting SGT sessions. This factor can be minimized in future by microteaching sessions among the tutors where more experienced faculty can guide junior tutors for improved teaching methods.

\section{Conclusion}

Students were satisfied with the small group sessions introduced in the department of Oral Medicine and Radiology. Further studies are needed to assess the students' perception of the integrated curriculum across undergraduate years in other subjects in dentistry. Conclusively it cab be said that, for a successful small group discussion, all the participants must be prepared for active listening, to take part in active discussions, share knowledge and skills for in-depth understanding of the topic.

\section{References}

1. Mona M. Soliman, Sami Abdulaziz Alnassar . Student perception of small group teaching in first year MBBS at King Saud University Medical College Saudi Arabia. PakJ Med Sci 2011;27(5):963-66.

2. Sarah Edmunds, George Brown. Effective small group learning: AMEE Guide No. 48 2010;32:715-26.

3. Dr Yvonne Steinert. Student perceptions of effective small group teaching. Medical Education 2004;38(3):286-93.

4. Nasir Aziz, Rabail Nasir, Abdus Salam. Students' Perception of Small Group Teaching: A Cross Sectional Study. Middle East J Family Med 2008;6(5)37-9.

5. Yvonne Steinert. Twelve tips for effective small-group teaching in the health Professions. Med Teacher 1996;18(3).

6. Krishna Prakash Joshi1, Suhasini Padugupati, M. Robins. Assessment of educational outcomes of small group discussion versus traditional lecture format among undergraduate medical students Int J Community Med Public Health 2018;5(7):2766-69 .

7. Mejbah Uddin Ahmed, Shusmita Roy.Small Group Teaching- An Update. Bangladesh J Med Microbiol 2014;08(02):28-31.

8. Sultan Ayoub Meo. Basic steps in establishing effective small group teaching sessions in medical schools. Pak J Med Sci 2013;29(4).

9. Dr Helena Ferris. The Use of Small Group Tutorials as an Educational Strategy in Medical Education. International Journal of Higher Education 2015;4(2).

10. Henry Walton. Small group methods in Medical Teaching. Med Educ 1997,31:459-64.

11. Sudheendra Kulkarni, Chandrakanth Chillarge. Evaluation of Small Group Discussion as a Teaching Method in Microbiology for Second Year medical Students in Brims Bidar: A Pilot Study. Int J Current Microbiol Applied Sci 2015;4(9):1001-03.

Feedback (Modified from Soliman et $\mathrm{al}^{1}$ )

\section{Annexure}

Likert scale was used to obtain student's perception.

(Strongly Agree, Agree, Can't say, Disagree, Strongly disagree)

This information will be kept confidential and will be used for only academic purpose.(Tick only one option)

1. The Small Group Teaching(SGT)session objectives were made clear to me

2. The location and facilities were suitable for the conduction of SGT sessions

3. Topics/Cases chosen for SGT were appropriate.

4. The sessions were thought provoking.

5. The sessions led me to self directed learning.

6. The sessions led me to identify my learning needs

7. SGT facilitates my participation in the teaching learning process.

8. SGT faculty were enthusiastic about the process

9. The faculty provided us lot of information

10. The faculty delivered a mini-lecture.

11. Faculties maintained discussion on track.

12. What I learned in these sessions was important and will be of use to me in the future.

13. These SGT improve my ability to think and problem solving skills.

14. These activities improved my ability to communicate effectively.

15. Overall, I was satisfied with the quality of these sessions.

16. Give your suggestions to improve the process of SGT

17. What did you like about SGT? 